Technical Note

\title{
Optimization of image quality in pulmonary CT angiography with low dose of contrast material
}

\author{
Abed Al Nasser AssI ${ }^{1,2, a}$, Ali ABU ARRA ${ }^{2}$ \\ ${ }^{I}$ Department of medical imaging, Arab American University, Jenin, Palestine \\ ${ }^{2}$ Department of medical imaging, An-Najah University, Nablus, Palestine \\ ${ }^{a}$ E-mail address: abed.assi@aauj.edu
}

(received 8 January 2017; revised 27 March 2017; accepted 10 May 2017)

\begin{abstract}
Aim: The aim of this study was to compare objective image quality data for patient pulmonary embolism between a conventional pulmonary CTA protocol with respect to a novel acquisition protocol performed with optimize radiation dose and less amount of iodinated contrast medium injected to the patients during PE scanning.

Materials and Methods: Sixty- four patients with Pulmonary Embolism (PE) possibility, were examined using angio-CT protocol. Patients were randomly assigned to two groups: A (16 women and 16 men, with age ranging from 19-89 years) mean age, 62 years with standard deviation 16; range, 19-89 years) - injected contrast agent: $35-40 \mathrm{ml}$. B (16 women and 16 men, with age ranging from 28-86 years) - injected contrast agent: $70-80 \mathrm{ml}$. Other scanning parameters were kept constant. Pulmonary vessel enhancement and image noise were quantified; signal-to-noise ratio (SNR) and contrast-to-noise ratio (CNR) were calculated. Subjective vessel contrast was assessed by two radiologists in consensus. Result: A total of 14 cases of PE (22\%) were found in the evaluated of subjects (nine in group A, and five in group B). All PE cases were detected by the two readers. There was no significant difference in the size or location of the PEs between the two groups, the average image noise was 14 HU for group A and 19 HU for group B. The difference was not statistically significant $(\mathrm{p}=0.09)$. Overall, the SNR and CNR were slightly higher on group B (24.4 and 22.5 respectively) compared with group A (19.4 and 16.4 respectively), but those differences were not statistically significant ( $p=0.71$ and $p=0.35$, respectively).

Conclusion and Discussion: Both groups that had been evaluated by pulmonary CTA protocol allow similar image quality to be achieved as compared with each other's, with optimize care dose for both protocol and contrast volume were reduced by $50 \%$ in new protocol comparing to the conventional protocol.
\end{abstract}

Key words: pulmonary embolism; signal to noise ratio; contrast to noise ratio; contrast volume; image quality.

\section{Introduction}

Multidetector computed tomography (MDCT) is the reference standard for the diagnosis of pulmonary embolism (PE) [1-4]. It is reported that the pulmonary embolism is the third most common acute cardiovascular disease, after myocardial infarction and stroke, and results in an estimated 200,000300,000 hospitalizations and 37,000-44,000 deaths per year in the United States [5]. Recent studies have shown the sensitivity of thin-slice "MDCT PE" to be $90-100 \%$ and the specificity to be $89-94 \%$ for the detection of pulmonary emboli to the level of the subsegmental arteries [4,6-9], using pulmonary angiography as the gold standard.

Several studies had revealed an overuse of CT angiography (CTA) to exclude PE, which increase in effective dose per patient [10-11], there is a need for reduction of radiation dose. Therefore the recent studies of pulmonary CTA have conducted important updates on optimizing protocols that restrict the amount of iodinated contrast media thus decreasing the risk of contrast medium-induced nephropathy particularly in people suffering from certain diseases such as diabetes and high blood pressure disease [12-14]. Thus, low radiation dose by using 128 MDCT scanning, low contrast medium volume CTA protocols may be potentially advantageous both to reduce radiation-derived risks and to prevent contrast medium-induced adverse event.

\section{Material and Methods}

\section{Subjects}

This study was held by Al Najah University Hospital in Nablus city, Palestine. Each individuals referred for pulmonary CTA for clinical suspicion of PE exam. To be eligible for the study patients had to have normal renal function. Patients with a personal history of allergy to iodinated contrast material, or impaired renal function (creatinine $>1.2 \mathrm{mg} / \mathrm{dl}$ ) were excluded from the study. Patients were divided in two groups: A (16 
women and 16 men, with age ranging from 19-89 years) injected contrast agent: $35-40 \mathrm{ml}$. B (16 women and 16 men, with age ranging from 28-86 years) - injected contrast agent: 70-80 ml.

\section{Pulmonary CTA protocol}

All pulmonary CTA examinations were performed using 128 MDCT system (Somatom Definition, Siemens Healthcare, Forchheim, Germany). For group A the acquisition parameter were: pitch $=1$, low contrast medium volume $=35-40 \mathrm{ml}$; flow rate $=4$; bolus tracking technique with Hounsfield attenuate threshold $=75-85 \mathrm{HU}$ and delay scan time after the contrast triggering in bolus-tracking technique is 3 second without breathing instruction before data acquisition. For group B the acquisition parameters were: pitch $=0.8$; contrast $=60-80 \mathrm{ml}$; flow rate $=4$; $\mathrm{HU}$ predefined attenuation threshold with delay scan time after the contrast triggering in bolus-tracking technique is 5 second with breathing instructions before data acquisition, bolus tracking with the region of interest placed in the main pulmonary artery. Pure, undiluted iodinated contrast medium was used for both groups (Iomeron 300), other parameters were kept constant for both groups (slice thickness is $3 \mathrm{~mm}, \mathrm{kVp}=100$ and care dose were selected for both group).

\section{Quantitative analysis of pulmonary CTA images}

All images were evaluated by two observers with 1 and 3 years of experience in CTA evaluation, blinded to the clinical history and pulmonary CTA protocol. Each study was subjectively classified as diagnostic or non-diagnostic by each reader. $3 \mathrm{~mm}$ slice thickness source images were employed for quantitative analysis.

The signal intensity for the central pulmonary arteries (SI vessels), given in HU, was defined as the attenuation measured by placing circular regions of interest (ROIs) in the centre of these vessels. The ROI size was adapted to the diameter of the vessel, reaching up to 80 to $90 \mathrm{~mm}^{2}$ in the central arteries. The main arteries were analysed by select the main pulmonary artery, left and right. In order to minimize bias, each artery was measured at three different locations. The mean of these values was used for further calculations.

The attenuation in the peripheral pulmonary arteries was ignored because unable to use the ROI method for assessing the signal intensity due to their small calibre.
To define the image noise, the standard deviations of $\mathrm{HU}$ measured in ROIs of at least $100 \mathrm{~mm}^{2}$ drawn in three different regions outside the patient body (middle, left, and right sides) were averaged.

The signal-to-noise ratio (SNR) was defined as SI vessel/ Noise. The contrast-to-noise ratio (CNR) was defined as (SI vessel - SI background)/Noise). These two parameters were calculated separately for the central pulmonary arteries.

For statistical analysis the SPSS software version 16.0 (SPSS Inc. Chicago, IL, USA) were applied and the independent sample t-test was employed to compare continuous variables. A two-sided $\mathrm{p}$-value $<0.05$ was considered to indicate a statistically significant difference.

\section{Results}

All subjects in this study with suspected PE underwent pulmonary CTA, without complications. No significant differences were observed in age, weight, and gender between individuals included in groups $\mathrm{A}$ and $\mathrm{B}$. Patient data are represent in Table 1. All studies were considered to have enough image quality to detect PE (Figure 1). A total of 14 cases of PE $(22.5 \%)$ were found in the evaluated of subjects (nine in group A, and five in group B). All PE cases were detected by the two readers. There was no significant difference in the size or location of the PEs between the two groups.

The average image noise was $14 \mathrm{HU}$ for group A and $19 \mathrm{HU}$ for group B. The difference was not statistically significant ( $p$ $=0.09$ ). Overall, the SNR and CNR were slightly higher on group B (24.4 and 22.5 respectively) compared with group A (19.4 and 16.4 respectively), but those differences were not statistically significant $(\mathrm{p}=0.71$ and $\mathrm{p}=0.35$, respectively).

Table 1. Structure of population, patient characteristics, signal intensity for the central pulmonary arteries and image quality in study groups data.

\begin{tabular}{lccc}
\hline \hline & Group A & Group B & P Value \\
\hline Male & 16 & 16 & \\
Female & 16 & 16 & \\
Weight (kg) & 80.4 & 85.6 & 0.82 \\
Vessel attenuation (HU) & 286 & 297 & 0.815 \\
Noise & 14 & 19 & 0.075 \\
SNR & 19.4 & 24.4 & 0.71 \\
CNR & 16.2 & 22.5 & 0.35 \\
\hline \hline
\end{tabular}




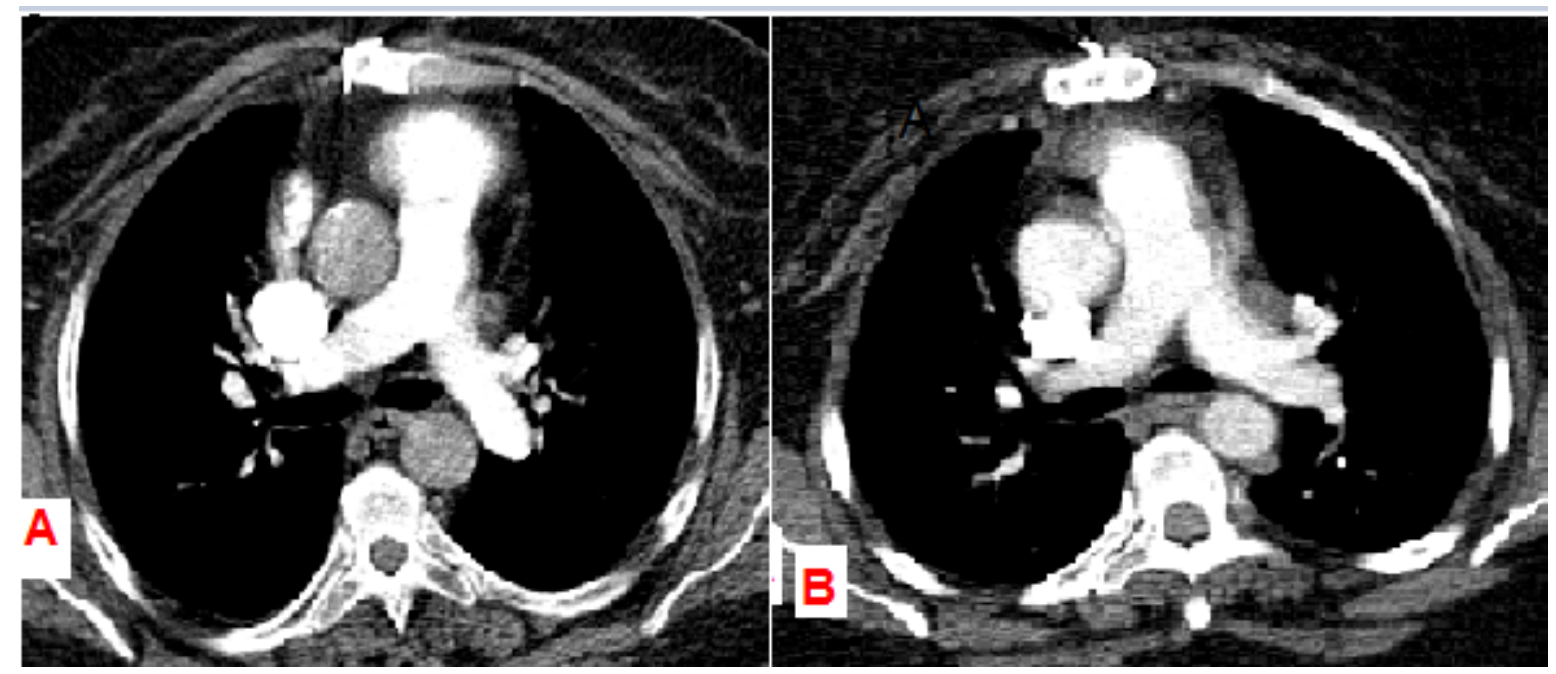

Figure 1. Exemplary $3 \mathrm{~mm}$-thick transverse CTPA images at $100 \mathrm{kVp}$ from the present patient collective. A. Image scan with protocol A (contrast volume 35-40 $\mathrm{cc}$ and pitch $=1$ ). B. Image scan with protocol B (contrast volume 80-100 cc and pitch= 0.8). For better comparison, the window level was set at $50 \mathrm{HU}$ and the window width at $350 \mathrm{HU}$ for all images. See the reduced beam hardening and streak artifact in image A.

\section{Discussion and Conclusions}

In the present study, the two groups were matched by gender, age, and weight, thus exclude possible variations between the groups in the vessel enhancement secondary to a different body habitus. Conversely, volume, concentration, injection rate, and duration of iodinated contrast medium administration are factors directly associated with pulmonary artery enhancement. As shown, the reduction in acquisition times achieved with the newest CT equipment avoids the need for long-duration contrast medium administration protocols, as the injection duration critically affects both the magnitude and timing of contrast enhancement [15-24]. This study used 128 MDCT Siemens machine during pulmonary scanning it managed to reduce injection period to less than $60 \%$ during CT scanning and this in turn reduce the size of the contrast medium to less than $50 \%$ of the value used in the classical pulmonary scanning protocol.

The most relevant finding of this study is that in individuals average weighing $80 \mathrm{~kg}$, a pulmonary CTA protocol with relatively small volume of iodinated contrast medium $(35 \mathrm{ml})$ results in pulmonary CT angiograms with lower injection period than using a conventional pulmonary CTA protocol (Figure 1). The novel protocol proposed here yielded a diagnostic confidence of PE that was not significantly different from the standard protocol, but using 50\% less intravenous contrast medium. This optimization of a standard pulmonary CTA protocol may have been achievable this is due to the latest technology, such as thin collimation and shortened study time provided by the new MDCT equipment, the use of automatic initiation of the examination by the bolus tracking technique, and the infusion of a saline flush immediately following the contrast medium which increase peak aortic enhancement through pushing into the cardiovascular system contrast material that otherwise would be left in the injection tubing. The saline flush also minimizes streak artifact from dense contrast which increases SNR of the pulmonary CTA $[25,26]$.

It is known that both normal and CT pulmonary angiography involve the injection of intravenous radiographic contrast under pressure and this injection may develop cardiopulmonary arrest after contrast injection [6]. The reduction of the amount of the contrast to $50 \%$ undoubtedly will reduce the likelihood of cardiac arrest, thus in turn will improve the use of CTA in diagnostic PE in a large scale. The reduction of the amount of contrast injection will also reduce beam hardening and streak artifact (see Figure 1), obscuring vessels especially in the area of the superior vena cava during the scan.

On order to spare participants from the double dose exposure, data existing in the database in the hospital (for previously used CTA screening protocol) were compared with results obtained for the new protocols, that use reduction of the contrast medium volume combined with high injection rate. This may influence the final results. In order to avoid any bias during comparing between two scanning protocols, the authors recommend the data to be collected for the same subjects on both protocols.

\section{References}

[1] Remy-Jardin M, Pistolesi M, Goodman LR, et al. Management of suspected acute pulmonary embolism in the era of CT angiography: a statement from the Fleischner Society. Radiology. 2007;245(2):315-329. 
[2] Perrier A, Roy PM, Sanchez O, et al. Multidetector-row computed tomography in suspected pulmonary embolism. N Engl J Med. 2005;352:1760-1788.

[3] Righini M, Le Gal G, Aujesky D, et al. Diagnosis of pulmonary embolism by multidetector CT alone or combined with venous ultrasonography of the leg: a randomised non-inferiority trial. Lancet. 2008;371(9621):1343-1352.

[4] Stein PD, Fowler SE, Goodman LR, et al. Multidetector computed tomography for acute pulmonary embolism. N Engl J Med. 2006; 354:2317-2327.

[5] Horlander KT, Mannino DM, Leeper KV. Pulmonary embolism mortality in the United States, 1979-1998: an analysis using multiple-cause mortality data. Arch Intern Med. 2003;163(14):1711-1717.

[6] Blachere H, Latrabe V, Montaudon M, et al. Pulmonary embolism revealed on helical CT angiography: comparison with ventilationperfusion radionuclide lung scanning. AJR Am J Roentgenol. 2000;174(4):1041-1047.

[7] Coche E, Verschuren F, Keyeux A, et al. Diagnosis of acute pulmonary embolism in outpatients: comparison of thin-collimation multi- detector row spiral CT and planar ventilation-perfusion scintigraphy. Radiology. 2003;229(3):757-765.

[8] Mayo JR, Remy-Jardin M, Muller NL, et al. Pulmonary embolism: prospective comparison of spiral CT with ventilation-perfusion scintigraphy. Radiology. 1997;205(2):447-452.

[9] Remy-Jardin M, Remy J, Baghaie F, et al. Clinical value of thin collimation in the diagnostic workup of pulmonary embolism. AJR Am J Roentgenol. 2000;175(2):407-411.

[10] O'Neill J, Murchison JT, Wright L, et al. Effect of the introduction of helical CT on radiation dose in the investigation of pulmonary embolism. Br J Radiol. 2005;78(925):46-50.

[11] O'Neill JM, Wright L, Murchison JT. Helical CTPA in the investigation of pulmonary embolism: a 6-year review. Clin Radiol. 2004;9(9):819-825.

[12] Holmquist F, Hansson K, Pasquariello F, et al. Minimizing contrast medium doses to diagnose pulmonary embolism with 80-kVp multidetector computed tomography in azotemic patients. Acta Radiol. 2009;50(2):181-193.

[13] Holmquist F, Nyman U. Eighty-peak kilovoltage 16-channel multidetector computed tomography and reduced contrast-medium doses tailored to body weight to diagnose pulmonary embolism in azotaemic patients. Eur Radiol. 2006;16(5):1165-1176.

[14] Szucs-Farkas Z, Kurmann L, Strautz T, et al. Patient exposure and image quality of low-dose pulmonary computed tomography angiography: comparison of 100- and 80-kVp protocols. Invest Radiol. 2008;43(12):871-876.

[15] Bae KT. Peak contrast enhancement in CT and MR angiography: when does it occur and why? Pharmacokinetic study in a porcine model. Radiology. 2003;227(3):809-816.

[16] Han JK, Kim AY, Lee KY, et al.. Factors influencing vascular and hepatic enhancement at CT: experimental study on injection protocol using a canine model. J Comput Assist Tomogr, 2000;24(3):400-406.

[17] Schoellnast H, Deutschmann HA, Berghold A, et al. MDCT angiography of the pulmonary arteries: influence of body weight, body mass index, and scan length on arterial enhancement at different iodine flow rates. AJR Am J Roentgenol. 2006;187(4):1074-1078.

[18] Dean PB, Violante MR, Mahoney JA. Hepatic CT contrast enhancement: effect of dose, duration of infusion, and time elapsed following infusion. Invest Radiol. 1980;15(2):158-161.

[19] Heiken JP, Brink JA, McClennan BL, et al. Dynamic contrast-enhanced CT of the liver: comparison of contrast medium injection rates and uniphasic and biphasic injection protocols. Radiology. 1993;187(2):327-331.

[20] Chambers TP, Baron RL, Lush RM. Hepatic CT enhancement. I. Alterations in the volume of contrast material within the same patients. Radiology. 1994;193(2):513-517.

[21] Kopka L, Rodenwaldt J, Fischer U, et al. Dual-phase helical CT of the liver: effects of bolus tracking and different volumes of contrast material. Radiology. 1996;201(2):321-326.

[22] Awai K, Hiraishi K, Hori S. Effect of contrast material injection duration and rate on aortic peak time and peak enhancement at dynamic CT involving injection protocol with dose tailored to patient weight. Radiology. 2004;230(1):142-150.

[23] Bae KT, Heiken JP. Scan and contrast administration principles of MDCT. Eur Radiol. 2005;15(suppl 5):E46-E59.

[24] Erturk SM, Ichikawa T, Sou H, et al. Effect of duration of contrast material injection on peak enhancement times and values of the aorta, main portal vein, and liver at dynamic MDCT with the dose of contrast medium tailored to patient weight. Clin Radiol. 2008;63(3):263-271.

[25] Dorio PJ, Lee Jr FT, Henseler KP, et al. Using a saline chaser to decrease contrast media in abdominal CT. AJR Am J Roentgenol. 2003;180:929-934.

[26] Haage P, Schmitz-Rode T, Hübner D, et al. Reduction of contrast material dose and artifacts by a saline flush using a double power injector in helical CT of the thorax. AJR Am J Roentgenol. 2000;174:1049-1053. 